\title{
EFFECT OF BODY MASS INDEX ON BLOOD PRESSURE AND LIPID PROFILE
}

\author{
Deepa Manuel ${ }^{1}$
}

${ }^{1}$ Assistant Professor, Department of Physiology, Karuna Medical College.

ABSTRACT
BACKGROUND
The importance of maintaining a healthy BMI is known all over the globe. Obesity causes Heart diseases, increase the potential for
Type 2 Diabetes Mellitus, increased risk of Stroke, Carcinomas, Hypertension and Hypercholesterolaemia. Our study emphasises
the result of other studies done on the same topic.

\section{MATERIALS AND METHODS}

This study was conducted in PSG Hospitals by taking Body Mass Index, Systolic-Diastolic BP and Lipid Profile from case sheets of Master Health Checkup. We took 153 subjects with normal BP, 153 subjects with abnormal BMI and compared the systolic and diastolic BP in the 2 groups using unpaired ' $t$ ' test. In the same manner Lipid profile was also compared in the normal BMI group and abnormal BMI group using unpaired ' $\mathrm{t}$ ' test.

\section{RESULTS}

This study shows a positive relationship between BMI, Hypertension and Lipid profile. In other words, we found that abnormal BMI is having a positive role in the pathogenesis of Hypertension and Hyperlipidaemia.

\section{CONCLUSION}

This study supports the results of other studies done on BMI. Healthy lifestyle modifications and maintaining a normal BMI can prevent Hypertension, Hypercholesterolaemia and its complications to some extent.

\section{KEYWORDS}

Hypertension, Body Mass Index, Lipid Profile.

HOW TO CITE THIS ARTICLE: Manuel D. Effect of body mass index on blood pressure and lipid profile. J. Evolution Med. Dent. Sci. 2017;6(83):5789-5792, DOI: 10.14260/jemds/2017/1256

BACKGROUND
Due to industrialisation and urbanisation, the standard of living continues to rise, particularly in developed countries. This has led to weight gain and obesity, which are posing a threat to the health of citizens. Obesity is perhaps the most prevalent form of malnutrition in developing countries, both among adults and children. Studies have demonstrated that obesity is related to elevated systolic blood pressure (SBP) and diastolic blood pressure (DBP) elevation, dyslipidaemia, diabetes, etc. ${ }^{1}$

Obesity and hypertension are both major public health problems all over the world. Results from the Framingham study have shown that high blood pressure ${ }^{2}$ and overweight ${ }^{3}$ are both independent risk factors for cardiovascular disease. Hypertension is one of the most common obesity-related complications and about 30\% hypertensive individuals can be classified as being obese. 4

So many studies conducted in many countries showed the increased BMI is related with hypertension and altered lipid profile. Jay S Kaufman ${ }^{5}$ et al showed the relationship between $\mathrm{BP}$ and BMI in lean population. Isabel Christina ${ }^{6}$ and Britto et al

'Financial or Other Competing Interest': None.

Submission 28-07-2017, Peer Review 03-10-2017,

Acceptance 09-10-2017, Published 16-10-2017.

Corresponding Author:

Dr. Deepa Manuel,

Assistant Professor

Staff Quarters, Karuna Medical College,

Vilayodi, Chittur,

Palakkad.

E-mail: deepamanuel@gmail.com

DOI: $10.14260 /$ jemds $/ 2017 / 1256$

(c) $(7)$ showed that childhood obesity as a risk factor for cardiovascular risk factors in adulthood like hypertension, dyslipidaemia, type 2 diabetes and insulin resistance.

Our study shows the effect of BMI on systolic-diastolic BP and mean arterial pressure.

A number of metabolic consequences of obesity have been proposed as the blood pressure-elevating mechanism. ${ }^{2}$ Increasing weight has been shown to increase salt retention and insulin resistance is proposed by some to be a cause of hypertension; adipose tissue produces substantial amounts of angiotensin which being a vasoconstrictor elevates BP. Physical activity can also have a substantial effect on blood pressure. 5

Obesity is a medical condition, in which excess body fat has accumulated to the extent that it may have an adverse effect on health. It is defined by body mass index (BMI) and further evaluated in terms of fat distribution via the waist-hip ratio and total cardiovascular risk factors. Obesity is a condition of abnormally increased body fat resulting from increased energy intake relative to energy expenditure. The prevalence of obesity is rapidly rising worldwide and obesity is a leading nutrition-related disorder. Overweight adolescents have increased risk of hypertension, heart disease and diabetes. ${ }^{7}$ BMI is calculated by dividing the subject's mass by the square of his or her height, typically expressed in $\mathrm{kg} / \mathrm{mt}^{2}$

As Asian populations develop negative health consequences at a lower BMI than Caucasians, some nations have redefined obesity. The Japanese have defined obesity as any BMI greater than 26, while China uses a BMI of greater than $28 .{ }^{8} \mathrm{On}$ an average obesity reduces life expectancy by six to seven years, ${ }^{9}$ a BMI of 30 - 35 reduces life expectancy by two-to-four years, ${ }^{4}$ while severe obesity $(\mathrm{BMI}>40)$ reduces 
life expectancy by 10 years. ${ }^{10}$ In this study, BMI more than 27 is taken as abnormal BMI.

Diabetes is associated with a greater risk of morbidity and mortality from cardiovascular disease (CVD) and heart disease is the leading cause of death among people with diabetes. Diabetes mellitus is a chronic disease that requires long-term medical attention, both to limit the development of its devastating complications and manage them when they occur. It is more common amongst developed countries where affluent and overweight individuals live longer than in under developed countries. Dyslipidaemia is a wellrecognised and modifiable risk factor for cardiovascular diseases, which is currently a leading cause of morbidity and mortality world-wide. Dyslipidaemia is common in DM, as both insulin deficiency and resistance affects enzymes and pathways of lipid metabolism. Diabetic dyslipidaemia is characterised by raised triglycerides, low high-density lipoprotein, raised apo-B and small dense low-density lipoprotein particles. It may be present at the diagnosis of type $2 \mathrm{DM}$ and it is a component of the metabolic syndrome. The pathogenesis of heart disease in diabetes is complex, but serum lipids are frequently abnormal and likely contribute to the risk of coronary artery disease. Lipids and lipoproteins are well known risk factors for ischaemic heart disease. Elevated levels of triglyceride, cholesterol and LDL are documented as risk factors for atherogenesis. ${ }^{11}$

Hypertension is a chronic cardiac medical condition, in which the systemic arterial blood pressure is elevated. About $90 \%-95 \%$ of cases are categorised as "primary hypertension," which means high blood pressure with no obvious medical cause. ${ }^{5}$

Persistent hypertension is one of the risk factors for stroke, myocardial infarction, heart failure and arterial aneurysm and is a leading cause of chronic kidney failure. Moderate elevation of arterial blood pressure leads to shortened life expectancy. ${ }^{12}$ Dietary and lifestyle changes can improve blood pressure control and decrease the risk of associated health complications, although drug treatment may prove necessary in patients for whom lifestyle changes prove ineffective or insufficient.

Lipid profile, also known as coronary risk panel or lipid panel, is the collective term given to the estimation of typically total cholesterol, high-density lipoprotein cholesterol, low-density lipoprotein cholesterol and triglycerides are used to assess risk of coronary heart disease.

\section{Objective}

In this study, we have checked whether there is any role of abnormal BMI in the pathogenesis of Hypertension and Hypercholesterolaemia.

\section{MATERIALS AND METHODS}

This study was conducted in PSG Institute of Medical Sciences and Research after getting clearance from Ethics Committee. It is a Descriptive study. After getting ethical clearance from ethical committee of PSG Institutions, we collected data from the Master health check-up case sheets like Height, Weight, Casual blood pressure and Lipid profile. Study included those subjects who were between 45 - 55 to exclude systolic hypertension in elderly; 153 subjects were in the normal BMI group and 153 subjects were in abnormal BMI group. Body mass index was calculated by the formula- weight in kilogram divided by height in meter square. Lipid profile was also collected which included Total Cholesterol, Triglycerides, HDL Cholesterol and LDL Cholesterol.

There were about 356 patients who were in the normal BMI group and 343 were in abnormal BMI group, which matched our criteria. Since normal BMI and abnormal BMI groups were almost equal in this study, we have taken the sample size of normal BMI and abnormal BMI as almost equal that is 153 . We selected 153 in normal BMI group and 153 in abnormal BMI group by simple random sampling.

We selected the subjects by the exclusion criteria by excluding the patients over 55 years of age. We included patients who were under antihypertensive medication and those who were under hypolipidaemic drugs, which has become a confounding factor. The sample size estimation was also done at conveniences.

To demonstrate the relationship between BMI and Hypertension, the study group was divided into normal BMI group, BMI less than 25 and abnormal BMI group. Body Mass Index above 27 was taken as abnormal. Systolic BP and Diastolic BP and Mean Arterial Pressure were compared in the two groups using unpaired ' $\mathrm{t}$ ' test using SPSS software version 22. P value was calculated and value $<.05$ was taken as statistically significant. Mean Arterial Pressure was calculated by formula Diastolic pressure plus one-third the pulse pressure.

To demonstrate the relationship between BMI and Lipid profile, the study group was divided into normal BMI and abnormal BMI group. Total cholesterol, Triglycerides, HDL and LDL cholesterol was compared in the two groups using unpaired ' $\mathrm{t}$ ' test.

Study by convenient sampling technique since the duration of the study was short duration. The sample size was selected by convenience sampling technique.

\section{RESULTS}

1. Comparison between BMI and Systolic Blood Pressure.

\begin{tabular}{|c|c|c|c|}
\hline & Mean & Standard Deviation & P value \\
\hline Normal BMI & 116.19 & 3.17 & \multirow{2}{*}{$<.05$} \\
\hline Increased BMI & 120.67 & 13.59 & \\
\hline \multicolumn{3}{|r|}{${ }^{*}$ means statistically significant } \\
\hline
\end{tabular}

Mean of systolic BP in normal BMI group was 116.19 with a standard deviation of 3.17. Mean in abnormal BMI group was 120.67 and standard deviation was 13.59 , p value equals $<.05$

2. Comparison of BMI and Diastolic BP.

\begin{tabular}{|c|c|c|c|}
\hline & Mean & Standard Deviation & P value \\
\hline Normal BMI & 74.7 & 4.74 & \multirow{2}{*}{$<.05^{*}$} \\
\hline Abnormal BMI & 76.81 & 6.54 & \\
\hline \multicolumn{4}{|c}{$*$ means statistically significant } \\
\hline
\end{tabular}

Mean of diastolic BP in normal BMI group was 74.7 with a standard deviation of 4.74. Mean in abnormal BMI group was 76.81 with a standard deviation of 6.54. P value was less than .05

3. Comparison of Mean Arterial Pressure in normal BMI and abnormal BMI group. 
$P$ value equals $<.05$

$\mathrm{i}$ - BMI is increased BMI

$\mathrm{n}$ - $\mathrm{BMI}$ is normal BMI

\begin{tabular}{|c|c|c|}
\hline & Mean & Standard Deviation \\
\hline N BMI & 83.33 & 5.62 \\
\hline I BMI & 85.88 & 6.98 \\
\hline
\end{tabular}

\section{Comparison between BMI and Systolic and Diastolic BP}

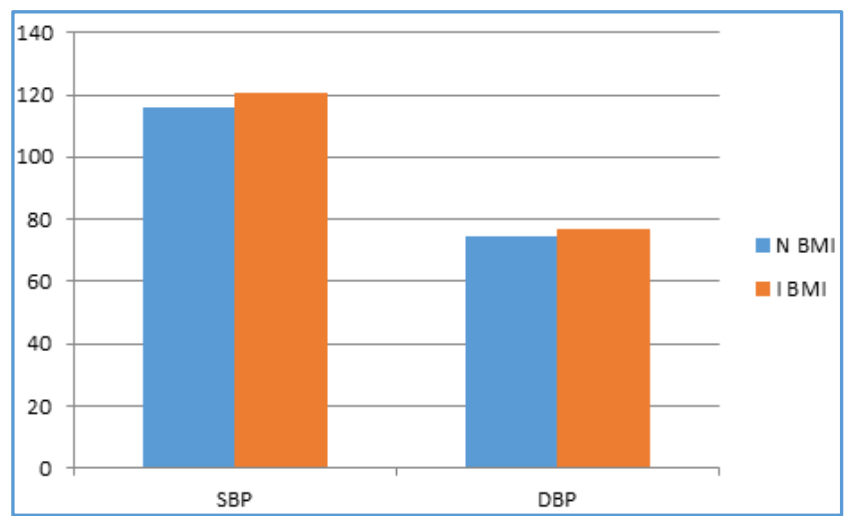

Comparison between BMI and Mean Arterial Pressure

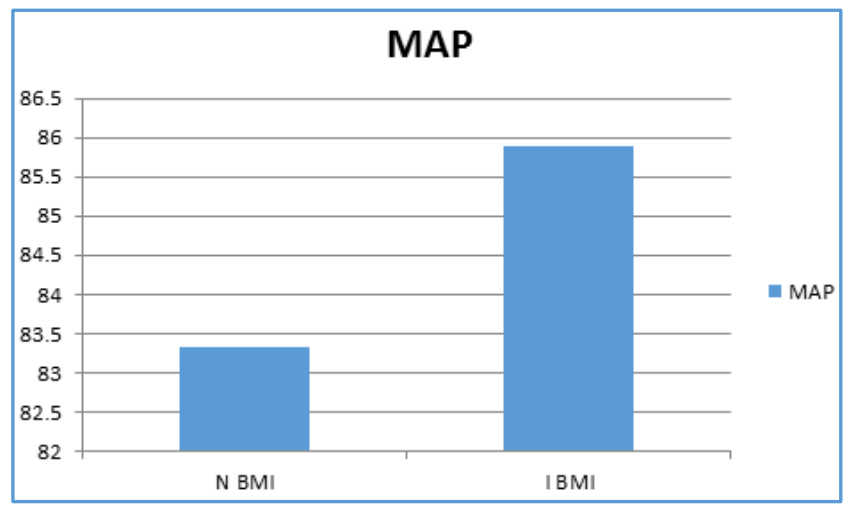

4. Comparison of Serum Cholesterol, Triglyceride, HDL Cholesterol, LDL Cholesterol in normal BMI group and abnormal BMI group.

\begin{tabular}{|c|c|c|c|c|}
\hline Parameter & Group & Mean & $\begin{array}{c}\text { Standard } \\
\text { Deviation }\end{array}$ & $\begin{array}{c}\text { P } \\
\text { value }\end{array}$ \\
\hline $\begin{array}{c}\text { Serum } \\
\text { Cholesterol }\end{array}$ & Normal BMI & 190.99 & 17.53 & \multirow{2}{*}{$<.05^{*}$} \\
\cline { 2 - 4 } Triglycerides & Increased BMI & 199.27 & 20.57 & \\
\cline { 2 - 4 } & Normal BMI & 140.27 & 14.02 & \multirow{2}{*}{$0.05^{*}$} \\
\hline $\begin{array}{c}\text { Indreased BMI } \\
\text { Cholesterol }\end{array}$ & 145.21 & 16.27 & $>$ \\
\cline { 2 - 4 } & Normal BMI & 42.57 & 10.55 & $>05^{\mathrm{NS}}$ \\
\hline $\begin{array}{c}\text { LDL } \\
\text { Cholesterol }\end{array}$ & Normal BMI & 114.54 & 9.02 & $<.05^{*}$ \\
\cline { 2 - 4 } & Increased BMI & 134.27 & 14.97 & \\
\hline \multicolumn{4}{|c}{$\begin{array}{c}\text { *means statistically significant } \\
\text { NS means not significant }\end{array}$} \\
\hline
\end{tabular}

Graphical representation of Lipid profile in normal and abnormal BMI group.
Graphical Representation of Lipid Profile in Normal and Abnormal BMI Group

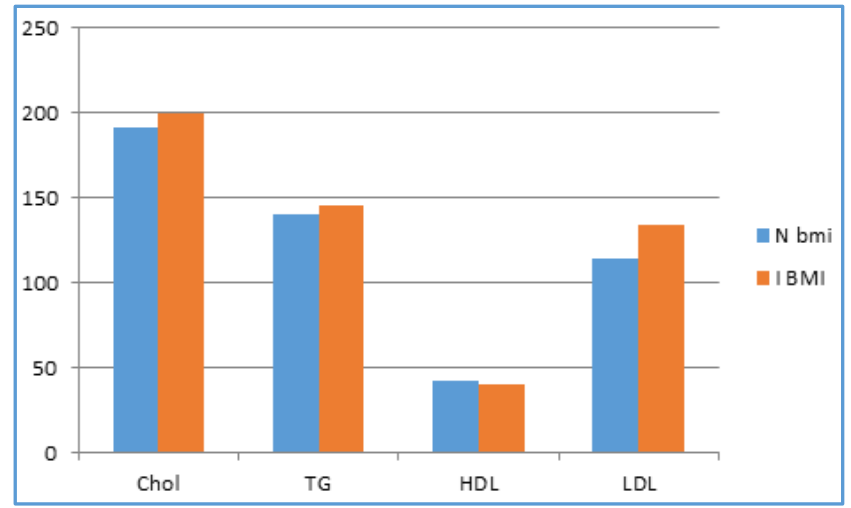

This graph clearly shows that Lipid profile is elevated in abnormal BMI group and HDL cholesterol has got no relationship with BMI. P value was 0.2676

\section{DISCUSSION}

Our study clearly shows the effect of BMI on systolic and diastolic blood pressure. Both systolic and diastolic BP was elevated in abnormal BMI group.

This study supports the findings of other studies. Ilse L Mertens and Luc Van Gaal ${ }^{13}$ et al demonstrated the effect of non-pharmacological means to reduce the BP. Nonpharmacological methods include weight reduction, exercises, low sodium intake, moderate alcohol intake, cessation of smoking and stress management. They suggested that moderate weight reduction will reduce Blood Pressure.

Isabel Christina and Britto ${ }^{6}$ et al showed a significant association of weight gain and abdominal obesity with blood pressure in adolescents.

Our study also showed a significant association of body mass index with lipid profile. Serum cholesterol, Triglyceride and LDL cholesterol showed a positive correlation with BMI. But HDL cholesterol does not show any association with BMI.

The association between measures of body mass and blood pressure has been extensively documented, usually with body mass index $\left(\mathrm{kg} / \mathrm{m}^{2}\right)$ as the measure of relative weight. Despite the consistency with which this correlation is observed, mechanistic explanations for the phenomenon are still being debated and no biological model of the process has been established. Epidemiological investigations have been hindered by several factors. The association however consistent is rather modest in magnitude and large sample sizes are therefore required in order to make estimates with any degree of certainty. Furthermore, the use of BMI has been questioned because percent body fat, absolute fat mass and body fat distribution or other relevant biological quantities may not be linearly related to BMI across the entire range of possible values or across different population subgroups. ${ }^{4}$ Finally, the effect of treatment in many populations truncates the distribution of blood pressures and therefore reduces the correlation that would be observed between BMI and blood pressure in untreated settings. ${ }^{14}$

This study stresses the importance of reducing the body weight in management of Hypertension and Hypercholesterolaemia. Thus, hypertension and hypercholesterolaemia has become a preventable cause of death across the globe. Hypertension is an important 
causative factor for heart diseases and stroke. Hypercholesterolaemia is an important cause for coronary vascular disease.

Study done by Droyvold et al shows that increase or decrease in BMI is associated with increase and decrease in systolic and diastolic blood pressure in adults. This further shows that by maintaining a normal BMI, we can control hypertension to some extent.

Obese children are at approximately a 3 -fold higher risk for hypertension than non-obese children. In addition, the risk of hypertension in children increases across the entire range of body mass index (BMI) values and is not defined by a simple threshold effect. 15

Consuming a diet low in fat and cholesterol and being aerobically fit predicted lower BMI, which together resulted in increases in high-density lipoprotein cholesterol and decreases in triglycerides and low-density lipoprotein cholesterol. Being physically active predicted greater aerobic fitness. ${ }^{16}$

Cardiovascular diseases are associated with risk factors such as obesity and dyslipidaemia, which if present during infancy could continue throughout adult life. High serum cholesterol HDL level is associated with longevity as evidence suggests. Low HDL cholesterol level is associated with an increased cardiovascular risk, particularly if serum cholesterol and triglycerides are also elevated. The most common primary lipid disorder familial combined hyperlipidaemia, which is an autosomal dominant condition occurring in $1 / 200$ people could not affect the results of our study with its low incidence. The most common dyslipidaemia of obesity is associated with increased triglyceride levels, decreased HDL levels and abnormal LDL cholesterol composition. Accepted risk factors for CVD are elevated LDL and decreased HDL. ${ }^{17}$

Reducing the body weight throughout life is important to reduce morbidity and mortality and to ensure quality of life and life expectancy. Our study shows that by maintaining normal body mass index, we can control hypertension and hypercholesterolaemia to an extent which will protect the individual from cardiovascular diseases like coronary artery diseases and stroke.

\section{Limitation of the Study}

Due to short duration of study, convenience sampling technique was followed, thus sampling size was also calculated by convenience. The results of the study cannot be generalised due to the potential bias resulting from the sampling technique and sample size estimation.

\section{CONCLUSION}

This study supports the results of other studies done on BMI. Healthy lifestyle modifications and maintaining a normal BMI can prevent hypertension, hypercholesterolaemia and its complications to some extent.

\section{REFERENCES}

[1] Dua S, Bhuker M, Sharma P, et al. Body mass index relates to blood pressure among adults. $\mathrm{N}$ Am J Med Sci 2014;6(2):89-95.
[2] Kannel WB. Blood pressure as a cardiovascular risk factor: prevention and treatment. JAMA 1996; 275(20):1571-6.

[3] Hubert HB, Feinleib M, McNamara PM, et al. Obesity as an independent risk factor for cardiovascular disease: a 26-year follow-up of participants in the framingham heart study. Circulation 1983;67(5):968-77.

[4] MacMahon S, Cutler J, Brittain E, et al. Obesity and hypertension: epidemiological and clinical issues. Eur Heart J 1987;8(Suppl B):57-70.

[5] Kaufman JS, Asuzu MC, Mufunda J, et al. Relationship between blood pressure and body mass index in lean populations. Hypertension 1997;30(6):1511-6.

[6] Pierdomenico SD, Di Nicola M, Esposito AL, et al. Prognostic value of different indices of blood pressure variability in hypertensive patients. Am J Hypertens 2009;22(8):842-7.

[7] Choi JW, Pai SH, Kim SK. Association between total body fat and serum lipid concentrations in obese human adolescents. Ann Clin Lab Sci 2002;32(3): 271-8.

[8] Zhou BF. Predictive values of body mass index and waist circumference for risk factors of certain related diseases in Chinese adults--study on optimal cut-off points of body mass index and waist circumference in Chinese adults. Biomed Environ Sci 2002;15(1):8396.

[9] Peeters A, Barendregt JJ, Willekens F, et al. Obesity in adulthood and its consequences for life expectancy: a life-table analysis. Ann Intern Med 2003;138(1): 24-32.

[10] Whitlock G, Lewington S, Sherliker P, et al. Body-mass index and cause-specific mortality in 900000 adults: collaborative analyses of 57 prospective studies. Lancet 2009;373(9669):1083-96.

[11] Omotoye FE, Fadupin GT. Effect of body mass index on lipid profile of type 2 diabetes at an urban tertiary hospital in Nigeria. JDMS 2016;15(9):65-70.

[12] Guimarães ICB, de Almeida AM, Santos AS, et al. Blood pressure: effect of body mass index and of waist circumference on adolescents. Arquivos Brasileros de Cardiologia 2008;90(6).

[13] Mertens IL, Van Gaal LF. Overweight, obesity \& blood pressure: the effect of moderate weight reduction. Obes Res 2000;8(3):270-8.

[14] Droyvold WB, Midthjell K, Nilsen TI, et al. Change in body mass index and its impact on blood pressure: a prospective population study. Int J Obes (Lond) 2005;29(6):650-5.

[15] Sorof J, Daniels S. Obesity hypertension in children: a problem of epidemic proportions. Hypertension 2002;40(4):441-7.

[16] Cugnetto ML, Saab PG, Liabre MM, et al. Lifestyle factors, body mass index, and lipid profile in adolesccents. J Pediatr Psychol 2008;33(7):761-71.

[17] Meral G, Uslu A, Yozgatli AU, et al. Association of body mass index and lipid profiles in children. Open Journal of Pediatrics 2015;5(2):141-6. 